\title{
Herdabilidades para ganho de peso da desmama ao sobreano e perímetro escrotal ao sobreano e tendências genética e fenotípica para ganho de peso da desmama ao sobreano em bovinos Nelore-Angus
}

\author{
Arione Augusti Boligon ${ }^{1}$, Paulo Roberto Nogara Rorato², Tomás Weber ${ }^{3}$, Dionéia Magda \\ Everling $^{4}$, Jader Silva Lopes ${ }^{5}$
}

\author{
${ }^{1}$ Pós-Graduação em Genética e Melhoramento Animal - UNESP - Campus Jaboticabal. Bolsista CNPq \\ ${ }^{2}$ Departamento de Zootecnia - Universidade Federal de Santa Maria. \\ 3 Pós-graduação em Zootecnia - UFSM. Bolsista CAPES. \\ ${ }^{4}$ Mestre em Zootecnia. \\ 5 Graduação em Zootecnia - UFSM
}

\begin{abstract}
RESUMO - Com o objetivo de estimar herdabilidades para ganho de peso médio diário da desmama ao sobreano (GMDDS) e para perímetro escrotal ao sobreano (PES) e tendências genética e fenotípica para GMDDS, foram utilizados 47.668 registros de peso e de ganho de peso de uma população multirracial Nelore-Angus, coletados entre 1991 e 2001 em diversas regiões do Brasil. Os dados foram analisados pelo método REML e as estimativas das (co)variâncias foram obtidas por meio de um modelo animal, no qual foram considerados fixos os efeitos da composição racial do animal (obtida pela concatenação do percentual da raça Nelore do próprio animal, de seu pai e de sua mãe) e do grupo de contemporâneos pós-desmama (animais nascidos no mesmo rebanho, ano, época e pertencentes ao mesmo sexo e grupo de manejo) e, como aleatórios, os efeitos genético aditivo direto e residual. A herdabilidade para PES foi estimada utlizando-se o mesmo modelo, acrescido dos efeitos fixos do peso e da idade do animal ao sobreano (covariáveis). As médias para idade nas pesagens foram 215 e 528 dias para a desmama e o sobreano, respectivamente. A herdabilidade estimada para GMDDS foi $0,44 \pm 0,02$ e para PES, $0,22 \pm 0,08$. A tendência genética anual predita para GMDDS foi decrescente até 1996 e crescente a partir desse período. A tendência fenotípica anual foi de $9,4 \mathrm{~g} / \mathrm{dia} / \mathrm{ano}$
\end{abstract}

Palavras-chave: progresso genético, regressão, valor genético

\section{Heritabilities for post-weaning daily gain and scrotal circumference at yearling age and genetic and phenotypic trends for post-weaning daily gain in Nellore-Angus crossbreds}

\begin{abstract}
Data consisting of 47.668 records of a Nellore-Angus crossbred population, raised in several regions of Brazil, from 1991 to 2001, were used to estimate heritability for post-weaning daily gain (ADG) and for scrotal circumference at yearling age (SCY) using REML. Genetic and phenotypic trends for ADG were also estimated. The model used to estimate heritability and breeding values (BVs) for ADG included the fixed effects of breed composition (defined by the percentage of contribution of the Nellore breed of the animal and his parents) and the contemporary group after weaning (herd, year/season of birth, sex and management group) and the random additive genetic animal and residual effects. The same model, including the fixed effects of weight and age of the animal at yearling, as covariates, was used for SCY. The average ages at weaning and yearling were respectively 215 and 528 days. The heritability estimates for ADG and SCY were $0.44 \pm 0.02$ and $0.22 \pm 0.08$, respectively. The annual genetic trend for ADG decreased up to the year of 1996 and increased then on. The phenotypic trend was $9.4 \mathrm{~g} /$ day/year.
\end{abstract}

Key Words: breeding values, genetic progress, regression

\section{Introdução}

O cruzamento entre raças européias e zebuínas tem sido amplamente utilizado em nosso meio criatório com o objetivo de produzir animais com maior desempenho nas condições ambientais em que são explorados. De acordo com Trematore et al. (1998), é necessário identificar os cruzamentos com maior vigor híbrido e que combinam características econômicas desejáveis para diferentes tipos de manejo e regiões do país, pois a superioridade dos animais cruzados em relação aos puros depende, principalmente, do ambiente que lhes é fornecido, das 
raças utilizadas e do valor genético dos indivíduos acasalados.

Dessa forma, torna-se importante avaliar o desempenho dos diferentes grupos genéticos obtidos a partir do cruzamento de animais das raças Nelore e Angus, em várias condições de manejo e ambiente e identificar qual responde melhor em cada situação.

O ganho de peso no período pós-desmama permite avaliar o potencial genético do indivíduo, visto que esse potencial não é mais influenciado pelo efeito materno. Segundo Ferraz (1995), o sucesso de qualquer programa de melhoramento, seja aplicado a raças puras ou direcionado à melhoria de animais cruzados, depende da correta identificação dos valores genéticos aditivos desses animais.

Entre as características de fertilidade, o perímetro escrotal tem sido utilizado com vistas ao aprimoramento da eficiência reprodutiva dos rebanhos bovinos, em razão de sua herdabilidade ser de magnitude média a alta (Alencar et al., 1993; Cyrillo et al., 2001; Gianlorenço et al., 2003).

A herdabilidade de determinada característica é fundamental em programas de melhoramento genético, pois características de baixa herdabilidade, exceto quando a intensidade de seleção é alta, não apresentam respostas satisfatórias ao trabalho de seleção dos animais.

A separação da fração genética da ambiental e a determinação da importância relativa dos efeitos aditivos genéticos diretos são necessárias quando se pretende formular planos de melhoramento animal (Ferreira et al., 1999), principalmente na fase pósdesmama, quando esses efeitos têm grande influência na resposta do animal.

O acompanhamento da evolução genética dos rebanhos é prática indispensável para verificar se o programa de seleção está sendo efetivo. Uma das formas para se realizar esse acompanhamento é por meio do cálculo da tendência genética direta, que fornece o acréscimo ou decréscimo ocorrido em determinada característica ao longo dos anos. Segundo Euclides Filho et al. (1997), o estudo da tendência genética ao longo do tempo, além de permitir a avaliação do progresso genético alcançado, serve, principalmente, como elemento orientador para ações futuras.

No caso de populações multirraciais, o cálculo de tendência genética também possibilita detectar os grupos genéticos com melhor desempenho no período estudado, auxiliando na escolha dos melhores cruzamentos a serem utilizados em cada propriedade.

O objetivo neste estudo foi estimar os coeficientes de herdabilidade para o ganho de peso médio diário da des- mama ao sobreano (GMDDS) e para o perímetro escrotal ao sobreano (PES) e as tendências genética e fenotípica para GMDDS em bovinos Nelore-Angus provenientes de diferentes regiões do Brasil.

\section{Material e Métodos}

Foram utilizados registros de peso à desmama e ao sobreano, bem como de medidas do perímetro escrotal ao sobreano (PES), tomados no período de 1991 a 2001, de 47.668 filhos de 3.154 touros de uma população multirracial Nelore-Angus. Os dados foram coletados em 34 fazendas de diversas regiões do Brasil, participantes de um programa de melhoramento genético realizado visando à produção de genótipos (nova raça) adaptados às condições do Brasil Central, cujos reprodutores são aptos à monta natural, como método de reprodução básico. Esta simplificação do manejo reprodutivo, juntamente com a maior adaptabilidade, deve proprocionar maior rentabilidade ao pecuarista desta região. A distribuição dos dados no período estudado é apresentada na Figura 1.

A composição genética dos grupos raciais nos diferentes rebanhos participantes do programa de seleção varia de acordo com a região, as características e os objetivos de cada propriedade, visto que o objetivo do programa é produzir um genótipo (raça) adaptado ao meio. Em decorrência do grande número de grupos raciais e visando minimizar o efeito da heterose ocorrida nos diferentes tipos de acasalamentos, foi criada uma variável considerando a porcentagem da raça Nelore nos animais em estudo, em um total de 28 combinações, fornecidas pela interação da composição racial do pai e da mãe (Tabela 1).

O sistema de produção desta população multirracial iniciou com a inseminação de vacas selecionadas da raça

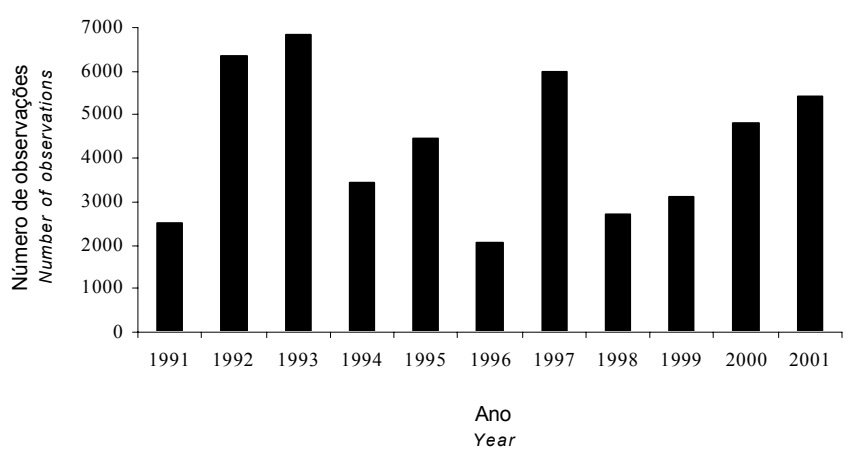

Figura 1 - Freqüência das observações, de acordo com o ano de nascimento.

Figure 1 - Frequency of observations by year of birth. 
Tabela 1 - Genótipo dos bezerros, com respectivos números de observações, e genótipos dos touros (pais) e das vacas (mães) nos rebanhos estudados, produtos dos cruzamentos entre as raças Angus (A) e Nelore $(N)$

Table 1 - Number of observations and genotypes of sires and dams of Angus (A) and Nellore $(N)$ crossbreds calves

\begin{tabular}{|c|c|c|c|}
\hline $\begin{array}{l}\text { Bezerro } \\
\text { Calf }\end{array}$ & $\begin{array}{l}\text { Número de observações } \\
\text { Number of observatios }\end{array}$ & $\begin{array}{l}\text { Touro (pai) } \\
\text { Sire }\end{array}$ & $\begin{array}{l}\text { Vaca (mãe) } \\
\text { Dam }\end{array}$ \\
\hline $3 / 16 \mathrm{~N}+13 / 16 \mathrm{~A}$ & 2.412 & $3 / 8 \mathrm{~N}+5 / 8 \mathrm{~A}$ & $0 \mathrm{~N}+1 \mathrm{~A}$ \\
\hline $9 / 32 \mathrm{~N}+23 / 32 \mathrm{~A}$ & 1.054 & $0 \mathrm{~N}+1 \mathrm{~A}$ & $9 / 16 \mathrm{~N}+7 / 16 \mathrm{~A}$ \\
\hline $9 / 32 \mathrm{~N}+23 / 32 \mathrm{~A}$ & 1.098 & $3 / 8 \mathrm{~N}+5 / 8 \mathrm{~A}$ & $3 / 16 \mathrm{~N}+13 / 16 \mathrm{~A}$ \\
\hline $5 / 16 \mathrm{~N}+11 / 16 \mathrm{~A}$ & 1.321 & $0 \mathrm{~N}+1 \mathrm{~A}$ & $5 / 8 \mathrm{~N}+3 / 8 \mathrm{~A}$ \\
\hline $5 / 16 \mathrm{~N}+11 / 16 \mathrm{~A}$ & 1.061 & $3 / 8 \mathrm{~N}+5 / 8 \mathrm{~A}$ & $1 / 4 \mathrm{~N}+3 / 4 \mathrm{~A}$ \\
\hline $5 / 16 \mathrm{~N}+11 / 16 \mathrm{~A}$ & 1.105 & $5 / 8 \mathrm{~N}+3 / 8 \mathrm{~A}$ & $0 \mathrm{~N}+1 \mathrm{~A}$ \\
\hline $11 / 32 \mathrm{~N}+21 / 32 \mathrm{~A}$ & 1.253 & $0 \mathrm{~N}+1 \mathrm{~A}$ & $11 / 16 \mathrm{~N}+5 / 16 \mathrm{~A}$ \\
\hline $11 / 32 \mathrm{~N}+21 / 32 \mathrm{~A}$ & 413 & $3 / 8 \mathrm{~N}+5 / 8 \mathrm{~A}$ & $5 / 16 \mathrm{~N}+11 / 16 \mathrm{~A}$ \\
\hline $11 / 32 \mathrm{~N}+21 / 32 \mathrm{~A}$ & 1.312 & $11 / 16 \mathrm{~N}+5 / 16 \mathrm{~A}$ & $0 \mathrm{~N}+1 \mathrm{~A}$ \\
\hline $3 / 8 \mathrm{~N}+5 / 8 \mathrm{~A}$ & 3.711 & $0 \mathrm{~N}+1 \mathrm{~A}$ & $3 / 4 \mathrm{~N}+1 / 4 \mathrm{~A}$ \\
\hline $3 / 8 \mathrm{~N}+5 / 8 \mathrm{~A}$ & 246 & $1 / 4 \mathrm{~N}+3 / 4 \mathrm{~A}$ & $1 / 2 \mathrm{~N}+1 / 2 \mathrm{~A}$ \\
\hline $3 / 8 \mathrm{~N}+5 / 8 \mathrm{~A}$ & 4.723 & $3 / 4 \mathrm{~N}+1 / 4 \mathrm{~A}$ & $0 \mathrm{~N}+1 \mathrm{~A}$ \\
\hline $13 / 32 \mathrm{~N}+19 / 32 \mathrm{~A}$ & 1.126 & $0 \mathrm{~N}+1 \mathrm{~A}$ & $13 / 16 \mathrm{~N}+3 / 16 \mathrm{~A}$ \\
\hline $13 / 32 \mathrm{~N}+19 / 32 \mathrm{~A}$ & 320 & $3 / 8 \mathrm{~N}+5 / 8 \mathrm{~A}$ & $7 / 16 \mathrm{~N}+9 / 16 \mathrm{~A}$ \\
\hline $7 / 16 \mathrm{~N}+9 / 16 \mathrm{~A}$ & 5.603 & $3 / 8 \mathrm{~N}+5 / 8 \mathrm{~A}$ & $1 / 2 \mathrm{~N}+1 / 2 \mathrm{~A}$ \\
\hline $7 / 16 \mathrm{~N}+9 / 16 \mathrm{~A}$ & 1.066 & $1 / 2 \mathrm{~N}+1 / 2 \mathrm{~A}$ & $3 / 8 \mathrm{~N}+5 / 8 \mathrm{~A}$ \\
\hline $1 / 2 \mathrm{~N}+1 / 2 \mathrm{~A}$ & 508 & $0 \mathrm{~N}+1 \mathrm{~A}$ & $1 \mathrm{~N}+0 \mathrm{~A}$ \\
\hline $1 / 2 \mathrm{~N}+1 / 2 \mathrm{~A}$ & 1.904 & $3 / 8 \mathrm{~N}+5 / 8 \mathrm{~A}$ & $5 / 8 \mathrm{~N}+3 / 8 \mathrm{~A}$ \\
\hline $1 / 2 \mathrm{~N}+1 / 2 \mathrm{~A}$ & 624 & $1 / 2 \mathrm{~N}+1 / 2 \mathrm{~A}$ & $1 / 2 \mathrm{~N}+1 / 2 \mathrm{~A}$ \\
\hline $1 / 2 \mathrm{~N}+1 / 2 \mathrm{~A}$ & 106 & $5 / 8 \mathrm{~N}+3 / 8 \mathrm{~A}$ & $3 / 8 \mathrm{~N}+5 / 8 \mathrm{~A}$ \\
\hline $9 / 16 \mathrm{~N}+7 / 16 \mathrm{~A}$ & 1.685 & $5 / 8 \mathrm{~N}+3 / 8 \mathrm{~A}$ & $1 / 2 \mathrm{~N}+1 / 2 \mathrm{~A}$ \\
\hline $19 / 32 \mathrm{~N}+13 / 32 \mathrm{~A}$ & 1.543 & $11 / 16 \mathrm{~N}+5 / 16 \mathrm{~A}$ & $1 / 2 \mathrm{~N}+1 / 2 \mathrm{~A}$ \\
\hline $21 / 32 \mathrm{~N}+11 / 32 \mathrm{~A}$ & 555 & $5 / 8 \mathrm{~N}+3 / 8 \mathrm{~A}$ & $11 / 16 \mathrm{~N}+5 / 16 \mathrm{~A}$ \\
\hline $11 / 16 \mathrm{~N}+5 / 16 \mathrm{~A}$ & 6.617 & $3 / 8 \mathrm{~N}+5 / 8 \mathrm{~A}$ & $1 \mathrm{~N}+0 \mathrm{~A}$ \\
\hline $3 / 4 \mathrm{~N}+1 / 4 \mathrm{~A}$ & 890 & $1 / 2 \mathrm{~N}+1 / 2 \mathrm{~A}$ & $1 \mathrm{~N}+0 \mathrm{~A}$ \\
\hline $3 / 4 \mathrm{~N}+1 / 4 \mathrm{~A}$ & 1.560 & $1 \mathrm{~N}+0 \mathrm{~A}$ & $1 / 2 \mathrm{~N}+1 / 2 \mathrm{~A}$ \\
\hline $13 / 16 \mathrm{~N}+3 / 16 \mathrm{~A}$ & 1.720 & $5 / 8 \mathrm{~N}+3 / 8 \mathrm{~A}$ & $1 \mathrm{~N}+0 \mathrm{~A}$ \\
\hline $13 / 16 \mathrm{~N}+3 / 16 \mathrm{~A}$ & 2.132 & $1 \mathrm{~N}+0 \mathrm{~A}$ & $5 / 8 \mathrm{~N}+3 / 8 \mathrm{~A}$ \\
\hline
\end{tabular}

Nelore com sêmen de touros selecionados da raça Angus. A seleção, para as raças puras e para as diferentes combinações genéticas, é feita com base no peso, mantendo-se os animais exclusivamente em pastagem, com manejo similar ao da região em que se situa o rebanho, no que se refere às condições de alimentação, sanidade e manejo, objetivando identificar animais mais eficientes biologicamente na utilização do alimento.

A estrutura dessa poppulação pode ser representada por uma pirâmide contendo, no ápice, um Núcleo Aberto de Seleção (NA), no qual encontram-se os animais das raças puras e os mestiços e para onde é destinado $1 \%$ das melhores fêmeas selecionadas no nível imediatamente inferior (Rebanhos Associados-RA); no NA também é permitida a entrada de reprodutores superiores, vindos de propriedades não participantes do programa e do nível RA. A base da pirâmide é constituída pelos rebanhos comerciais RC nos quais não é realizada seleção.

Para maior consistência, os grupos de contemporâneos com menos de cinco animais e touros com menos de cinco filhos, bem como os registros incompletos, foram previa- mente excluídos na elaboração dos arquivos. Foram também excluídos os registros de animais com GMDDS inferior a 103 g ou superior a 890 g. Os limites para exclusão foram obtidos por meio de 2,5 desvios-padrão, para mais ou para menos, em relação à média.

Os animais foram pesados à desmama (média de 215 dias) e ao sobreano (528 dias).

Para a estimativa dos componentes de variância e covariância utilizados para estimar os coeficientes de herdabilidade e predizer os valores genéticos (VG) para GMDDS, adotou-se um modelo estatístico que incluiu como fixos os efeitos da composição racial do animal (obtida pela concatenação do percentual da raça Nelore do próprio animal, de seu pai e de sua mãe) e do grupo de contemporâneos pós-desmama (animais nascidos no mesmo ano, estação e fazenda e pertencentes ao mesmo sexo e grupo de manejo) e, como aleatórios, os efeitos genético aditivo direto e residual.

A estimativa do coeficiente de herdabilidade para a característica perímetro escrotal ao sobreano (PES) foi feita utilizando-se o mesmo modelo, acrescido dos efeitos da idade e do peso do animal ao sobreano. 
O método de análise utilizado foi o da máxima verossimilhança restrita livre de derivada e o aplicativo, o MTDFREML (Boldman et al., 2001).

O modelo de análise adotado é descrito, matricialmente, como:

$$
\mathrm{y}=\mathrm{Xb}+\mathrm{Za}+\mathrm{e}
$$

em que: $\mathrm{y}=$ vetor das observações do caráter ganho de peso médio diário do desmame ao sobreano (GMDDS) e do perímetro escrotal; $\mathrm{X}=$ matriz de incidência associada aos efeitos fixos; $b=$ vetor de solução para os efeitos fixos de grupo de composição racial, grupo de contemporâneos e das covariáveis; $Z$ = matriz de incidência associada aos efeitos aleatórios; a = vetor de soluções para os efeitos aleatórios direto do animal; e = vetor dos resíduos.

Para o estudo da tendência genética direta, foi realizada a regressão ponderada das médias anuais dos valores genéticos para a característica de GMDDS sobre o ano de nascimento utilizando-se o procedimento REG (SAS, 2001).

\section{Resultados e Discussão}

A estimativa de herdabilidade direta para GMDDS foi de $0,44 \pm 0,02$, indicando que o valor fenotípico pode ser usado como estimador do valor genético aditivo direto. Conseqüentemente, espera-se obter ganho genético por meio da seleção para ganho de peso pós-desmama. A herdabilidade estimada nesse trabalho está de acordo com os resultados obtidos por Martins Filho et al. (1997), para a raça Gir, e por Balbé et al. (2003), para as raças Brangus e Angus. No entando, valores inferiores foram relatados por Euclides Filho et al. (1998) e Fernandes et al. (2001) para as raças Gir e Charolesa, respectivamente. Relato semelhante foi feito por Sarmento et al. (2003), em estudo com bovinos da raça Nelore. Esses autores concluíram que a seleção com base no ganho de peso no período de um ano até um ano e meio de idade pode promover considerável progresso genético. Porém, Mascioli et al. (2000), em estudo com animais da raça Canchim, concluíram que o ganho médio diário do nascimento à desmama é uma característica que responde bem à seleção, enquanto o ganho médio diário pós-desmama apresenta menor resposta.

A estimativa de herdabilidade para PES foi de $0,22 \pm$ 0,08 , semelhante à relatada por Everling et al. (2001), de 0,21, utilizando animais da mesma composição racial e mesmo modelo deste trabalho. Estimativas superiores às obtidas neste estudo foram relatadas por Bergmann et al. (1996), Ortiz Peña et al. (2001), Garnero et al. (2001) e Dias et al. (2003) para a raça Nelore e Alencar et al. (1993) e Silva et al. (2000) para a raça Canchim. A estimativa de herdabilidade para PES, neste estudo, também foi inferior à média dos trabalhos revisados por Koots et al. (1994), que observaram que as estimativas de herdabilidade foram maiores para dados não ajustados, intermediárias para dados ajustados para idade e menores quando houve ajuste para peso corporal. Comparando modelos para determinação de fatores de ajustes para perímetro escrotal, Ortiz Peña (1998) e Dias et al. (2003) encontraram estimativas de herdabilidades menores e aumento na variância residual para o modelo que incluiu o perímetro escrotal ajustado apenas para idade, em relação ao modelo que incluiu o perímetro escrotal ajustado para idade e peso, ressaltando, assim, a importância da correção do PES para a idade e o peso corporal. Estudos mais aprofundados do perímetro escrotal utilizando diferentes modelos deveriam ser realizados para observação das diferentes estimativas de herdabilidade, pois são raros os trabalhos na literatura que relacionam características de crescimento com perímetro escrotal em populações multirraciais.

A tendência genética direta para GMDDS no período de 1991 a 2001 é representada na Figura 2.

A tendência genética direta estimada para GMDDS foi decrescente até 1996, indicando ausência ou ineficiência de programas de seleção durante este período, e crescente a partir desse período e até o último ano estudado. Entretanto, o progresso genético aditivo estimado provavelmente está aquém do que poderia ser alcançado, considerando a variabilidade genética para esta característica nesta população.

A média do progresso genético direto estimado foi de 3,66 g/dia/ano para o período entre 1996 e 2001. Esse

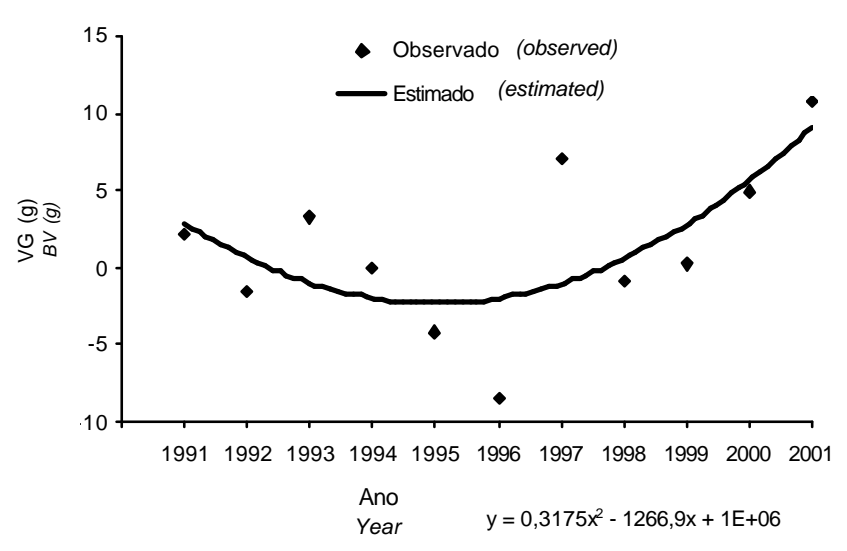

Figura 2 - Tendência genética para ganho de peso médio diário da desmama ao sobreano (GMDDS).

Figure 2 - Genetic trend for average daily gain from weaning to yearling (ADG). 
resultado é aproximadamente três vezes maior que o relatado por Balbé et al. (2003), que estimaram tendências genéticas diretas para GMDDS para a raça Angus e 37 vezes maior que o relatado por esses autores para a raça Brangus. Do mesmo modo, o resultado obtido neste estudo é superior ao relatado por Euclides Filho et al. (1998), em estudo com rebanhos da raça Gir, os quais observaram, como resultado da regressão das DEP para ganho de peso médio diário pósdesmama, o valor de $0,03 \mathrm{~g} / \mathrm{dia} / \mathrm{ano}$.

Baixos valores para tendência genética podem ser explicados pela atuação de efeitos ambientais ou pela pequena seleção direcional. Tendências genéticas próximas de zero e, até mesmo, negativas são comuns na literatura, principalmente quando resultam de avaliações realizadas com dados provenientes de rebanhos comerciais, cujos critérios de seleção não estão bem definidos e, principalmente, não são uniformes (Razook et al., 1993). Contudo, o valor obtido foi menor que o relatado por Martins Filho et al. (1997), que estimaram tendências genéticas de 360, 250 e 310 g/ano para animais das raças Gir, Guzerá e Nelore, respectivamente.

Na Figura 3 é descrita a tendência fenotípica, ou seja, a porção genética mais o efeito do meio ambiente, estimada em 9,4 g/dia/ano para GMDDS.

Estes resultados indicam que o progresso anual obtido pelos produtores no desempenho dos animais analisados neste estudo é conseqüência, quase que totalmente, da melhoria nas condições de meio ambiente, isto é, da adaptação dos genótipos aos ambientes em que estão sendo explorados, visto que a porção genética aditiva não está contribuindo de forma efetiva.

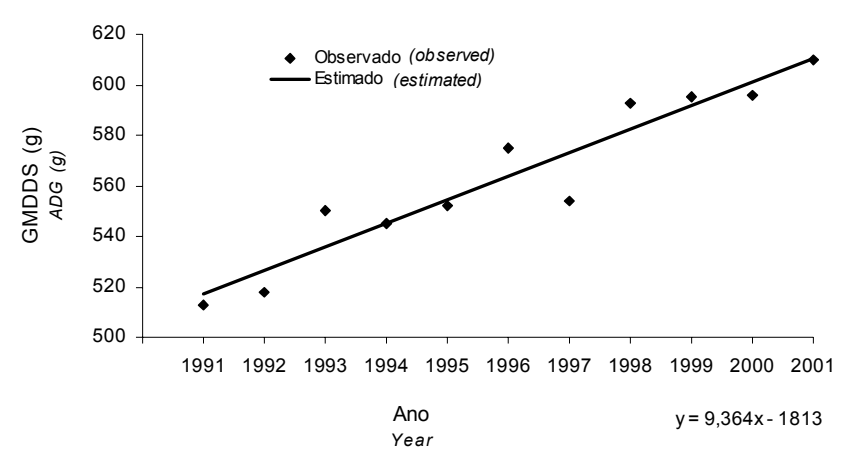

Figura 3 - Tendência fenotípica para ganho médio diário da desmama ao sobreano (GMDDS).

Figure 3 - Phenotypic trend for average daily gain from weaning to yearling ( $A D G)$.

\section{Conclusões}

As estimativas de herdabilidade para ganho de peso médio diário pós-desmama e perímetro escrotal ao sobreano sugerem que estas características apresentam considerável variação genética aditiva, sendo, portanto, passíveis de seleção direta.

Foi observado progresso fenotípico para GMDDS, provavelmente conseqüência de melhoria ambiental.

Mesmo obtendo progresso genético crescente a partir de 1996, torna-se evidente a necessidade da utilização de programas de melhoramento que possibilitem maior e melhor desempenho dos animais nas condições a que estão submetidos.

Os resultados indicam que mesmo rebanhos com alto progresso fenotípico podem apresentar pouco progresso genético, tornando-se de grande importância a realização de trabalhos nesta linha de pesquisa visando alcançar progressos genéticos e fenotípicos mais efetivos para o rebanho bovino do Brasil.

\section{Literatura Citada}

ALENCAR, M.M.; BARBOSA, P.F.; BARBOSA, R.T. et al. Parâmetros genéticos para peso e circunferência escrotal em touros da raça Canchim. Revista da Sociedade Brasileira de Zootecnia, v.22, n.4, p.572-583, 1993.

BALBÉ, D.D.; BOLIGON, A.A.; KIPPERT, C.J. et al. Tendência genética para ganho de peso médio diário entre a desmama e o sobreano nas raças Angus e Brangus. In: REUNIÃO ANUAL DA SOCIEDADE BRASILEIRA DE ZOOTECNIA, 40., 2003, Santa Maria. Anais... Santa Maria: Sociedade Brasileira de Zootecnia, 2003. (CD-ROM).

BERGMANN, J.A.G.; ZAMBORLINI, L.C.; PROCÓPIO, C.S.O et al. Estimativas de parâmetros genéticos do perímetro escrotal em animais da raça Nelore. Arquivo Brasileiro de Medicina Veterinária e Zootecnia, v.48, n.1, p.69-78, 1996.

BOLDMAN, K.H.; KREISE, L.A.; Van VLECK, L.D. et al. A manual for use for MTDFREML. A set of programs to obtain estimates of variance and covariances (DRAFT). Lincoln: Departament of Agriculture/Agricultural Research Service, 2001. $120 \mathrm{p}$.

CYRILlO, J.N.S.G.; RAZOOK, A.G.; FIGUEIREDO, L.A. et al. Estimativas de tendências e parâmetros genéticos do peso padronizado aos 378 dias de idade, mediads corporais e perímetro escrotal de machos Nelore de Sertãozinho, SP. Revista Brasileira de Zootecnia, v.30, n.1, p.56-65, 2001.

DIAS, L.T.; EL FARO, L.; ALBUQUERQUE, L.G. Estimativas de herdabilidade para perímetro escrotal de animais da raça Nelore. Revista Brasileira de Zootecnia, v.32, n.6, p.1878-1882, 2003 (supl. 2).

EUCLIDES FILHO, K.; SILVA, L.O.C.; ALVES, R.G.O. et al. Tendências genéticas de ganhos de peso pré e pós desmama na raça Gir. In: REUNIÃO ANUAL DA SOCIEDADE BRASILEIRA DE ZOOTECNIA, 35., 1998, Botucatu. Anais... São Paulo: SBZ/Gnosis, 1998 (CD-ROM) 17 par. Melhoramento Animal. MEL-123. 
EUCLIDES FILHO, K.; SILVA, L.O.C.; FIGUEIREDO, G.R. Tendências genéticas na raça Guzerá. In: REUNIÃO ANUAL DA SOCIEDADE BRASILEIRA DE ZOOTECNIA, 34., 1997, Juiz de Fora. Anais... Juiz de Fora: Sociedade Brasileira de Zootecnia, 1997. p.173.

EVERLING, D.M.; FERREIRA, G.B.B.; RORATO, P.R.N. et al. Estimativas de herdabilidade e correlação genética para características de crescimento na fase de pré-desmama e medidas de perímetro escrotal ao sobreano em bovinos Angus-Nelore. Revista Brasileira de Zootecnia, v.30, n.6, p.2002-2008, 2001 (supl.).

FERNANDES, H.D.; FERREIRA, G.B.; RORATO, P.R.N. et al. Tendência genética para ganho de peso pós-desmama na raça Charolês. In: REUNIÃO ANUAL DA SOCIEDADE BRASILEIRA DE ZOOTECNIA, 38., 2001, Piracicaba. Anais... Piracicaba: Sociedade Brasileira de Zootecnia, 2001. p.505-506.

FERRAZ, J.B.S. Sistema de avaliação de cruzamento e avaliação genética. In: CURSO SOBRE AVALIAÇÃO GENÉTICA EM BOVINOS DE CORTE, 1., 1995, Ribeirão Preto. Apostila... Ribeirão Preto: 1995. 20p.

FERREIRA, G.B.; MacNEIL, M.D.; Van VLECK, L.D. Variance components and breeding values for growth traits from different statistical models. Journal of Animal Science, v.77, p.2641-2650, 1999 .

GARNERO, A.V.; LÔBO, R.B.; BEZERRA, L.A.F. et al. Comparação entre alguns critérios de seleção para crescimento da raça Nelore. Revista Brasileira de Zootecnia, v.30, n.3, p.714-718, 2001.

GIANLORENÇO, V.K.; ALENCAR, M.M.; TORAL, F.L.B. et al. Herdabilidade e correlações genéticas de características de machos e fêmeas, em um rebanho bovino da raça Canchim. Revista Brasileira de Zootecnia, v.32, n.6, p.1587-1593, 2003 (supl. 1).

KOOTS, K.R.; GIBSON, P.J.; SMITH, C. et al. Analyses of published genetic parameter estimates for beef production traits. 1. Herdability. Animal Breeding Abstracts, v.62, n.5, p.309370, 1994.

MARTINS FILHO, R.; LOBO, R.N.B.; LIMA, F.A.M. et al. Parâmetros genéticos e fenotípicos de pesos e ganho em pesos de bovinos zebus no estado do Ceará. In: REUNIÃO ANUAL DA SOCIEDADE BRASILEIRA DE ZOOTECNIA, 34., 1997, Juiz de Fora. Anais... Juiz de Fora: Sociedade Brasileira de Zootecnia, 1997. p.248-250.
MASCIOLI, A.S.; EL FARO, L.; ALENCAR, M.M. et al. Estimativas de parâmetros genéticos e fenotípicos e análise de componentes principais para características de crescimento da raça Canchim. Revista Brasileira de Zootecnia, v.29, n.6, p.1654-1660, 2000.

ORTIZ PEÑA, C.D.O.; QUEIROZ, S.A.; FRIES, L.A. et al Comparação entre critérios de seleção de precocidade sexual e a associação destes com características de crescimento em bovinos Nelore. Revista Brasileira de Zootecnia, v.30, n.1, p.93-100, 2001 .

RAZOOK, A.G.; FIGUEIREDO, L.A.; BONILHA NETO, L.M. et al. Intensidades de seleção e respostas direta e correlacionadas em 10 anos de progênies de bovinos das raças Nelore e Guzerá selecionadas para peso pós-deamame. Boletim da Indústria Animal, v.50, n.2, p.147-163, 1993.

SARMENTO, J.L.R.; PIMENTA FILHO, E.C.; RIBEIRO, R.M.F. Efeitos ambientais e genéticos sobre ganho em peso diário de bovinos Nelore no estado da Paraíba. Revista Brasileira de Zootecnia, v.32, n.2, p.325-330, 2003.

STATISTICAL ANALYSIS SYSTEMS - SAS. User's guide: Version 6.11 ed. Cary: 2001.

SILVA, A.M.; ALENCAR, M.M.; FREITAS, A.R. et al. Herdabilidade e correlações genéticas para peso e perímetro escrotal de machos e características reprodutivas de crescimento de fêmeas, na raça Canchim. Revista Brasileira de Zootecnia, v.29, n.6, p.22232230, 2000 (supl. 2).

TREMATORE, R.L.; ALENCAR, M.M.; BARBOSA, P.F. et al. Estimativas de efeitos aditivos e heteróticos para características de crescimento em bovinos Charolês-Nelore. Revista Brasileira de Zootecnia, v.27, n.1, p.87-94, 1998. 